\title{
LA OFICINA MUNICIPAL DE CONSERVACIÓN Y REHABILITACIÓN DE LA CIUDAD HISTÓRICA DE SANTIAGO DE COMPOSTELA (GALICIA, ESPAÑA)
}

\author{
(THE MUNICIPAL CONSERVATION AND REHABILITATION OFFICE OF THE \\ HISTORIC CITY CORE OF SANTIAGO DE COMPOSTELA. GALICIA, ESPAÑA)
}

Javier Ramos, Fernando Ga Tobío, Idoia Camiruaga, Ángel Panero, Xose S. Allegue y Ramón Fdez. Hermida

\begin{tabular}{|c|c|}
\hline $\begin{array}{l}\text { OFICINA MUNICIPAL } \\
\text { DE CONSERVACIÓN } \\
\text { E REHABILITACIÓN } \\
\text { DA CIDADE HISTÓRICA. }\end{array}$ & \\
\hline Director Técnico: & - Javier Ramos \\
\hline Gestor: & - Fernando $\mathrm{G}^{\mathrm{a}}$. Tobío \\
\hline $\begin{array}{l}\text { Coordinadores de Proyectos } \\
\text { y Obras: }\end{array}$ & $\begin{array}{l}\text { - Idoia Camiruaga } \\
\text { Ángel Panero }\end{array}$ \\
\hline Coordinador Area de formación: & - Xose S. Allegue \\
\hline $\begin{array}{l}\text { Coordinador de la Sección } \\
\text { Técnica: }\end{array}$ & - Ramón Fdez Hermida \\
\hline Delineación: & $\begin{array}{l}\text { - Luis Gianzo } \\
\text { José A. Ga. Martínez }\end{array}$ \\
\hline Auxiliares: & $\begin{array}{l}\text { - } \text { M José Serantes }^{\text {Liana Ramos }} \\
\text { Luis Muñoz }\end{array}$ \\
\hline
\end{tabular}

RESUMEN

El casco histórico de Santiago de Compostela fue declarado por la UNESCO Bien Cultural de Interés Universal en el mes de diciembre de 1985. El Consorcio de la Ciudad de Santiago promueve, a través del Ayuntamiento de Santiago, la creación de una Oficina Técnica que se ocupe especificamente del diseño y desarrollo de los programas de rehabilitación de la Ciudad Histórica. A la espera de la aprobación definitiva del "Plan Especial de Protección y Rehabilitación de la Ciudad Histórica de Santiago de Compostela", la Oficina de Rehabilitación diseñó, y gestiona desde hace un año, el "Plan Puente de Rehabilitación Interior de Viviendas de la Ciudad Histórica" con el objetivo de mejorar las condiciones de vida de sus habitantes mediante la realización de obras de mejora y saneamiento de las instalaciones de electricidad y fontanería, asi como de los acabados interiores de las viviendas. El artículo presenta el modelo de Oficina, los programas diseñados y su funcionamiento desde que la Oficina Municipal de Conservación y Rehabilitación de la Ciudad Histórica fue creada en junio de 1994.

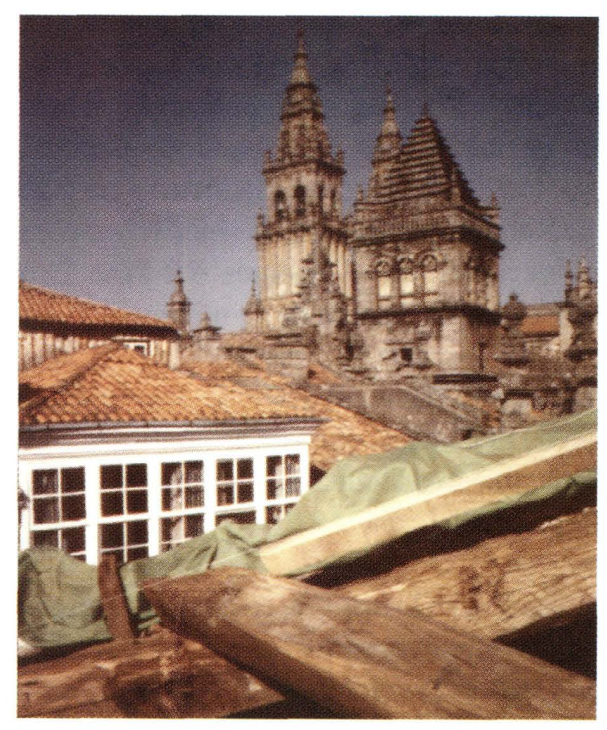

SUMMARY

UNESCO declared the historic core of Santiago de Compostela a Cultural Asset of Universal Interest in December, 1985. The City of Santiago Trust, through the Santiago de Compostela City Council, promotes the creation of a technical Office to be specifically in charge of designing and developing rehabilitation programmes for the Historic City Core. While waiting for the final approval of the "special Protection and Rehabilitation Plan for the Historic City Core of Santiago de Compostela", the Rehabilitation Office has designed and managed during one year the "Bridging Plan for Interior Rehabilitation of Dwellings in the Historic Core of Santiago de Compostela", with the purpose of improving the living conditions of its occupants by means improving and repairing works of electricity and plumbing installations, as well as of the interior finishes of such dwellings. The paper presents the Office model, the designed programmes and the performance there of since the Municipal-Office of Conservation and Rehabilitation of the Historic City Core was created in June, 1994. 


\section{Presentación}

Desde 1992 el Consorcio de la Ciudad promueve, a través del Concello, un conjunto de medidas de fomento y ayuda tendentes a garantizar la mejora de las condiciones de vida de los habitantes de la Ciudad Histórica y el mantenimiento de su conjunto edificado, reconocido como Patrimonio de la Humanidad.

El Consorcio de la Ciudad, en virtud del Convenio firmado el día 27 de abril de 1994, encomienda al Concello el establecimiento de las medidas oportunas para la ejecución de las actuaciones de rehabilitación de la Ciudad Histórica y que continúen la labor emprendida en 1992 en la que, con la colaboración ciudadana, se adecentaron los exteriores de los edificios del Centro Histórico como medida de protección de sus interiores, mejorando al tiempo su imagen urbana.

En junio de 1994, el Concello de Santiago contrata, mediante concurso público, los servicios de una empresa especializada para diseñar el organigrama, el método y las funciones de la Oficina Municipal de Conservación y Rehabilitación de la Ciudad Histórica (en adelante, la Oficina de Rehabilitación), cuyos objetivos generales figuraban ya recogidos en el Avance del Plan Especial de Protección y Rehabilitación de la misma.

La Oficina ocupa parte del Pazo de Vaamonde, sede del Consorcio de la Ciudad, una vez rehabilitado su interior y dotada de los medios técnicos adecuados a la función que desarrollará en adelante.

Como se observará a lo largo de este artículo, el criterio que ha guiado las actuaciones de la Oficina durante este año está basado en la conservación del Patrimonio de la Ciudad Histórica a través de la mejora de las condiciones de uso de los edificios que la conforman, consecuencia de las necesidades de sus habitantes y conforme a la orientación y determinaciones recogidas en el Avance del Plan Especial.

Definidas en éste las actuaciones que deben llevarse a cabo en el conjunto de la Ciudad Histórica, la Oficina actúa como órgano que encauza, aúna o requiere los esfuerzos de los ciudadanos para completar puntuales intervenciones cuando se considera el momento oportuno, en función de las carencias o deseos de los usuarios en cada ámbito de su actuación y de las intervenciones de recuperación urbana programadas en el Plan Especial

\section{2. Ámbito de actuación ${ }^{*}$}

El ámbito de actuación de la Oficina de Rehabilitación es el de la Ciudad Histórica, definido en el Plan General y con ligeras modificaciones en el Plan Especial.

${ }^{(*)}$ Datos extraídos de la información del Avance del Plan Especial.
Forman esteámbito 2.829 edificaciones, de las que 169 son dotacionales. Existen en él 6.717 viviendas (aproximadamente un $21,3 \%$ del parque municipal), y solamente un $14,3 \%$ ( 960 viviendas) se encuentran vacías.

En la Ciudad Histórica viven 17.401 personas, de las que 4.834 son transeúntes (principalmente estudiantes).

Los edificios son de una única vivienda en 1.181 casos (44\%), y de más de seis en 239 ocasiones (9\%).

Están en buen estado un 50\%, mientras que precisan obras de rehabilitación ligera o media un $38,5 \%$ y alta un $10,7 \%$ de los mismos, los considerados en mal estado.

Son de propiedadúnica el $83,1 \%$, mientras que pertenecen al régimen de comunidad el $14,9 \%$ de los mismos. Las instituciones poseen un $2 \%$ del parque edificatorio.

Se hallan en régimen de alquiler un $38 \%$ de las viviendas, y permanecen con renta antigua $600(24 \%$ de las viviendas alquiladas).

Entre las patologías más significativas se encuentran el mal estado de las cubiertas ( $9,8 \%$ de los edificios), el mal estado de la instalación eléctrica $(10,9 \%)$, los daños estructurale importantes $(8,6 \%)$ y los edificios con problemas de verticalidad $(2,6 \%)$

El tamaño de las viviendas se distribuye en:

\begin{tabular}{|c|c|c|}
\hline Superficie & $\begin{array}{c}\text { Número de } \\
\text { Viviendas }\end{array}$ & Porcentaje \\
\hline $0-40 \mathrm{~m}^{2}$ & 1.290 & $20,0 \%$ \\
\hline $41-70 \mathrm{~m}^{2}$ & 2.341 & $36,2 \%$ \\
\hline $71-100 \mathrm{~m}^{2}$ & 1.337 & $20,7 \%$ \\
\hline $101-150 \mathrm{~m}^{2}$ & 888 & $13,7 \%$ \\
\hline más de $150 \mathrm{~m}^{2}$ & 610 & $9,4 \%$ \\
\hline
\end{tabular}

Por último, los locales en la Ciudad Histórica son 1.857, de los que están ocupados 1.605 .

El $40,6 \%$ tienen un uso comercial y el $26,0 \%$ hostelero. El resto se reparten entre oficinas y servicios $(13,5 \%)$ y otras actividades $(19,9 \%)$.

En régimen de propiedad se encuentran el 50,5\%, mientras que mantienen un alquiler de renta antigua el $38,4 \%$.

\section{Estructura de la oficina}

Desde enero de 1995, en que sus plazas fueron cubiertas por concurso público, la Oficina de Re⿳亠厶⺝abilitación está compuesta por: 
Un Director Técnico. Coordinador de los diferentes programas de rehabilitación y de los recursos técnicos de la Oficina. Arquitecto.

Un Gestor. Coordinador de los aspectos administrativos de la rehabilitación. Economista.

Dos coordinadores de Proyectos y obras. Directores de los proyectos generados por los Programas de rehabilitación y supervisores de las obras. Arquitectos.

Un coordinador del Área de formación. Encargado de diseñar los Programas de educación y fomento de la Rehabilitación. Coordinador de la primera visita técnica de diagnóstico de las viviendas que se realiza cuando los inquilinos o propietarios solicitan la colaboración de la Oficina de Rehabilitación. Arquitecto.

Un Jefe de la Sección Técnica. Encargado de la coordinación del Área de producción de memorias y presupuestos. Encargado del mantenimiento del equipo informático. Técnico de grado medio.

Dos delineantes especializados en el manejo de programas informáticos. Formalización de documentaciones técnicas.

Dos auxiliares administrativas. Encargadas de la atención al público, tramitaciones de documentos y archivo.

Un conserje. Encargado también de la papelería, fax y fotocopias.

\section{Programas iniciales}

Para el diseño de los primeros programas se partió del diagnóstico que se formula en el Avance del Plan Especial sobre la Ciudad Histórica, según el cual "desde el punto de vista de su actual realidad urbanistica, la característica más destacable y que la singulariza entre el conjunto de ciudades históricas similares es, sin duda, su relativa, vigencia como realidad urbana, conservada todavia en gran medida en sus aspectos residenciales, funcionales, económicos y sociales".

No obstante, se constata asimismo la existencia de determinados fenómenos patológicos de carácter estructural, tales como "el avance del proceso de terciarización, el abandono del centro por sus habitantes tradicionales, el deterioro del patrimonio edificado y del ambiente urbano, (...) que se hacen patentes en distintos grados y con distinta intensidad, pero en todos los casos con una tendencia a la progresiva agravación".

Por ello, se consideró conveniente poner en marcha -antes de la aprobación definitiva del Plan Especial, pero plenamente incardinados en la estrategia de recuperación urba- na por él diseñada- el Programa Puente de Rehabilitación Interior de Viviendas y el Plan Piloto y, posteriormente, el Programa Puente de Ayudas a los Locales Comerciales.

Los Programas Puente se conciben como instrumentos de intervención inmediata, dirigidos a actuar sobre la generalidad de los inmuebles de la ciudad histórica con medidas de tipo remediador y encaminadas a frenar o contrarrestar los efectos más perniciosos de aquellos procesos detectados en el Avance del Plan Especial. Por su ámbito temporal, tienen vocación de transitoriedad, a la espera de la aprobación definitiva del Plan Especial, a partir de la cual se establecerá un marco de ayudas a la rehabilitación más estable y permanente.

El Plan Piloto tiene un contenido eminentemente estructural, dirigido a vehicular intervenciones singulares y espacialmente discretas y a coordinar una serie de actuaciones, en apoyo de las estrategias trazadas por el Plan Especial, y con un horizonte temporal de medio y largo plazo.

\subsection{El programa puente de rehabilitación interior}

Vigente desde el día 1 de noviembre de 1994, y concebido inicialmente para un período de duración de 6 meses, en cuanto al plazo de solicitud de ayudas, se consideró conveniente ampliarlo por 8 meses más, hasta el 31 de diciembre de 1995 , a la vista del elevado número de solicitudes que se producen.

Se configura como segunda fase del programa de rehabilitación, iniciado con el arreglo de las fachadas de la Ciudad Histórica y conforme a las orientaciones establecidas en el Avance del Plan Especial, en el que se establecen los criterios que deben encauzar la conservación de nuestro Patrimonio con la necesaria planificación, y conscientes de que esta conservación depende, en gran medida, del mantenimiento de los usos residenciales y de la mejora de las condiciones de vida de sus habitantes.

El Programa se instrumenta a través de la "Ordenanza de Ayudas a la Rehabilitación Interior de Viviendas de la Ciudad Histórica", que constituye la pieza normativa básica del mismo. En ella se establecen los tipos de ayudas a la rehabilitación, condiciones y procedimientos para acceder a ellas.

Esta Ordenanza fue diseñada por la Oficina de Rehabilitación y está destinada a resolver los problemas de las viviendas de la ciudad Histórica que carecen de los servicios sanitarios básicos, presentan instalaciones eléctricas o de fontanería en mal estado, o tienen serias deficiencias en los elementos comunes de la edificación (portal, escaleras, etc.), contribuyendo así a frenar el proceso de deterioro del patrimonio residenciałedificado y a fijar la población residente. 

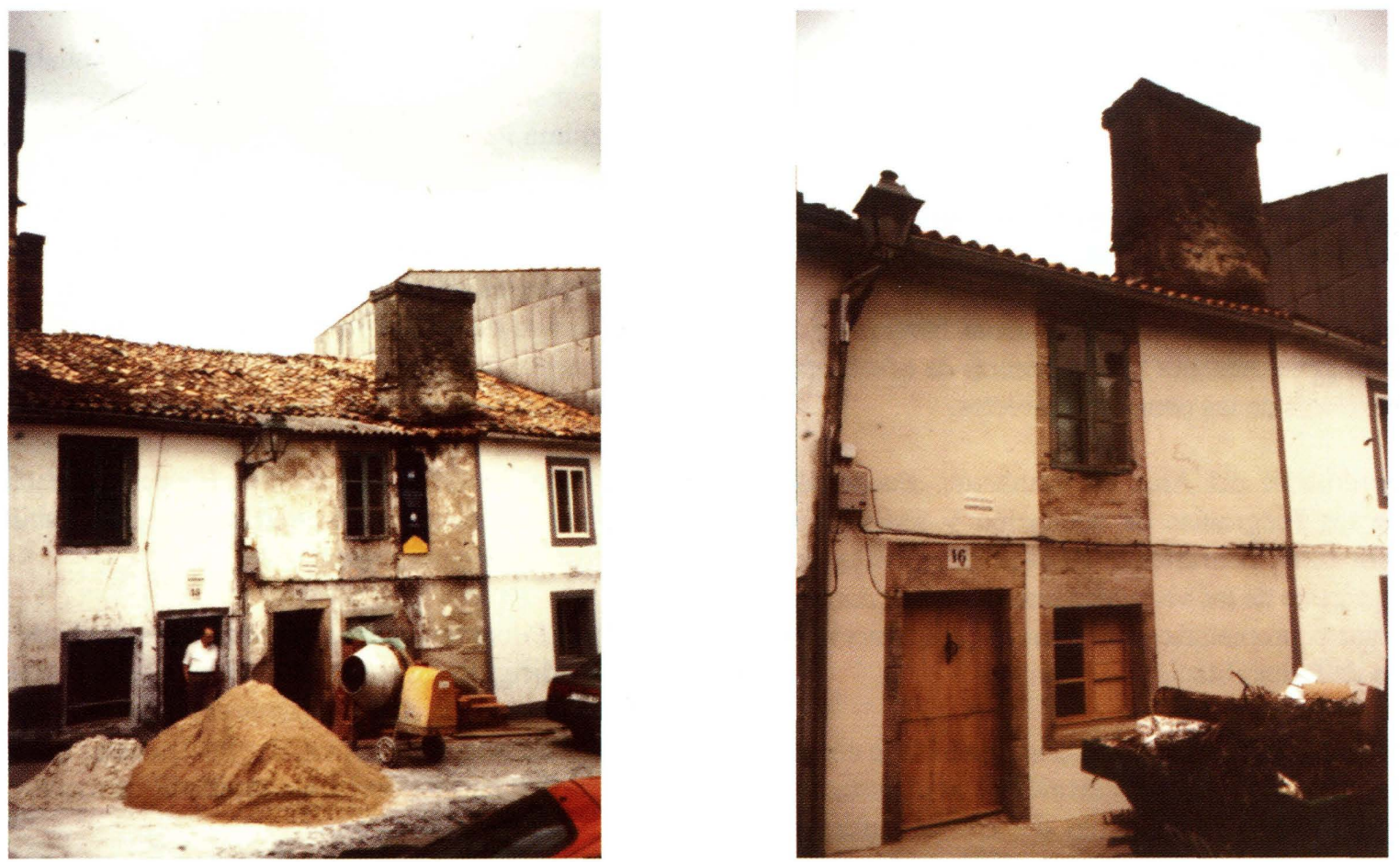

Las obras que con el control de la Oficina se realizan bajo la cobertura del "Plan Puente de Rehabilitación de Interiores", pretenden, fundamentalmente, mejorar las condiciones de habitabilidad de las viviendas históricas. Por ello, estas obras, son generalmente actuaciones sobre las instalaciones eléctricas y de fontanería y los acabados interiores de las viviendas. Sin embargo, no se pierde la oportunidad de realizar obras complementarias que mejoren la imagen urbana de los inmuebles. En las fotografias se puede contrastar el estado inicial y el estado casifinal de las obras realizadas en la vivienda situada en el bajo del $n^{\circ} 16$ de la calle del Espíritu Santo, donde además de las obras interiores se realizaron, de acuerdo con los propietarios, las obras complementarias de reparación de cubierta, fachada y carpinterias exteriores de planta baja.

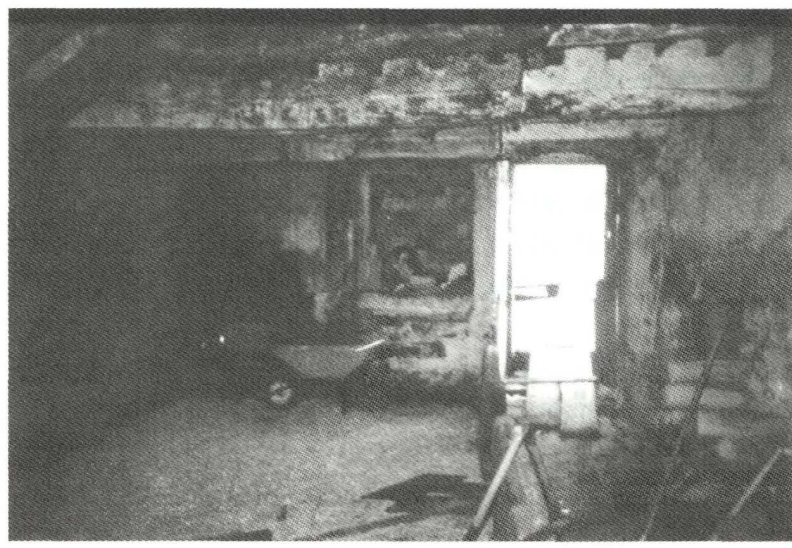

La Ordenanza fue aprobada definitivamente por el Pleno Municipal, en la sesión celebrada el día 3 de octubre de 1994, y publicada en el Boletín Oficial de la Provincia del día 29 del mismo mes.

Las subvenciones de este Programa se establecen sobre el presupuesto protegible de la obra, en función de los ingresos familiares y de la cuantía de la obra a realizar, para

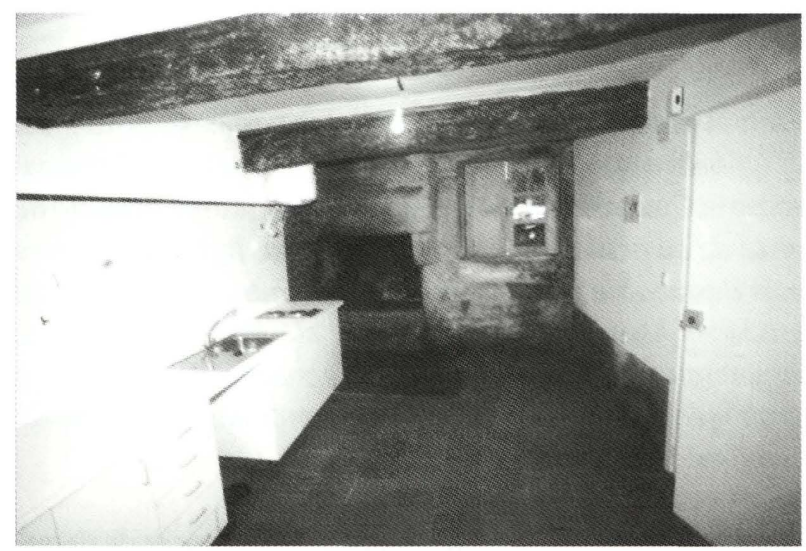

favorecer las situaciones económicamente débiles e incentivar la realización de obras convenientes para la conservación del edificio.

El sistema creado por la Oficina de Rehabilitación garantiza un control absoluto y riguroso no sólo del uso de la subvención concedida, sino también del planteamiento y ejecución de las obras realizadas. 

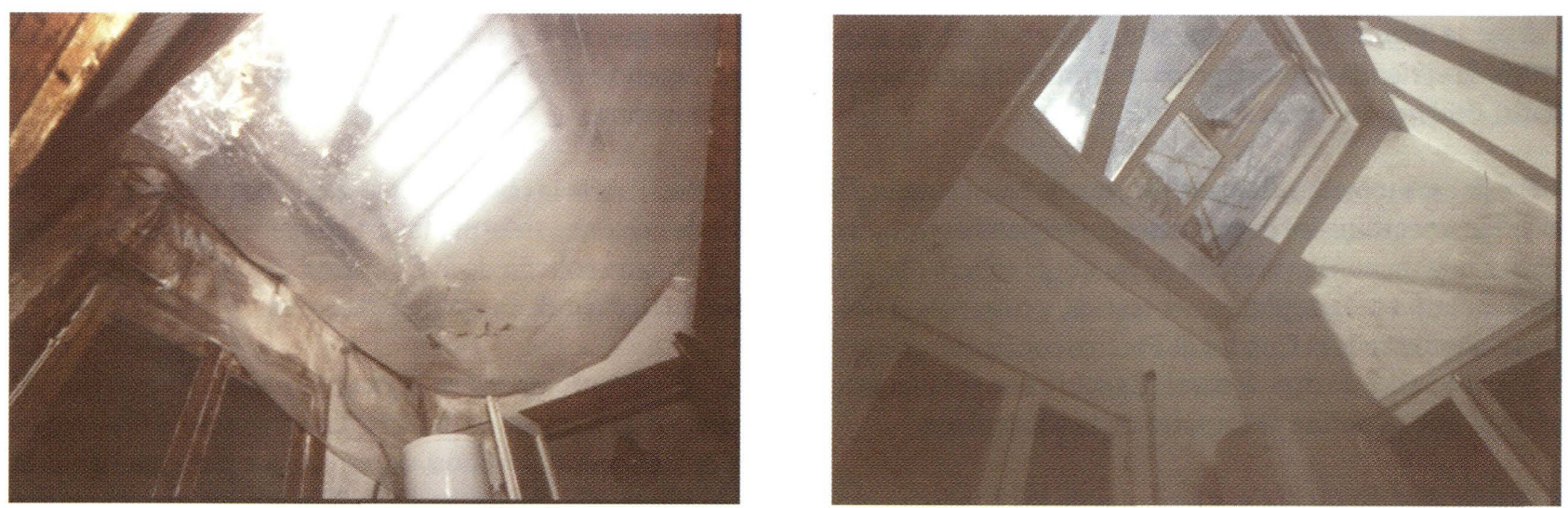

Estado inicial y final de la intervención sobre un lucernario de cubierta muy deteriorado. Al espacio que cubre el lucernario ventilan la cocina y el baño de la vivienda, por esta razón se instaló una ventana motorizada que permitiese controlar fácilmente el grado de aireación de la estancia. La carpintería es de aluminio lacado y los vidrios con cámara y luna stadip exterior.

El proceso para la concesión de una ayuda económica es el siguiente:

1. El particular solicita en la Oficina la visita de un técnico al lugar donde se pretenden realizar determinadas obras de su interés.

\section{Un arquitecto de la Oficina visita el edificio y asesora al}

usuario sobre la posibilidad de realizar las obras por él solicitadas, asesorándole sobre otros aspectos que contribuirían también a mejorar sus condiciones de vida, al tiempo que contribuyen a conservar el edificio. De esta visita de inspección técnica queda un informe en el expediente.

3. El particular solicita oficialmente la ayuda, presentando
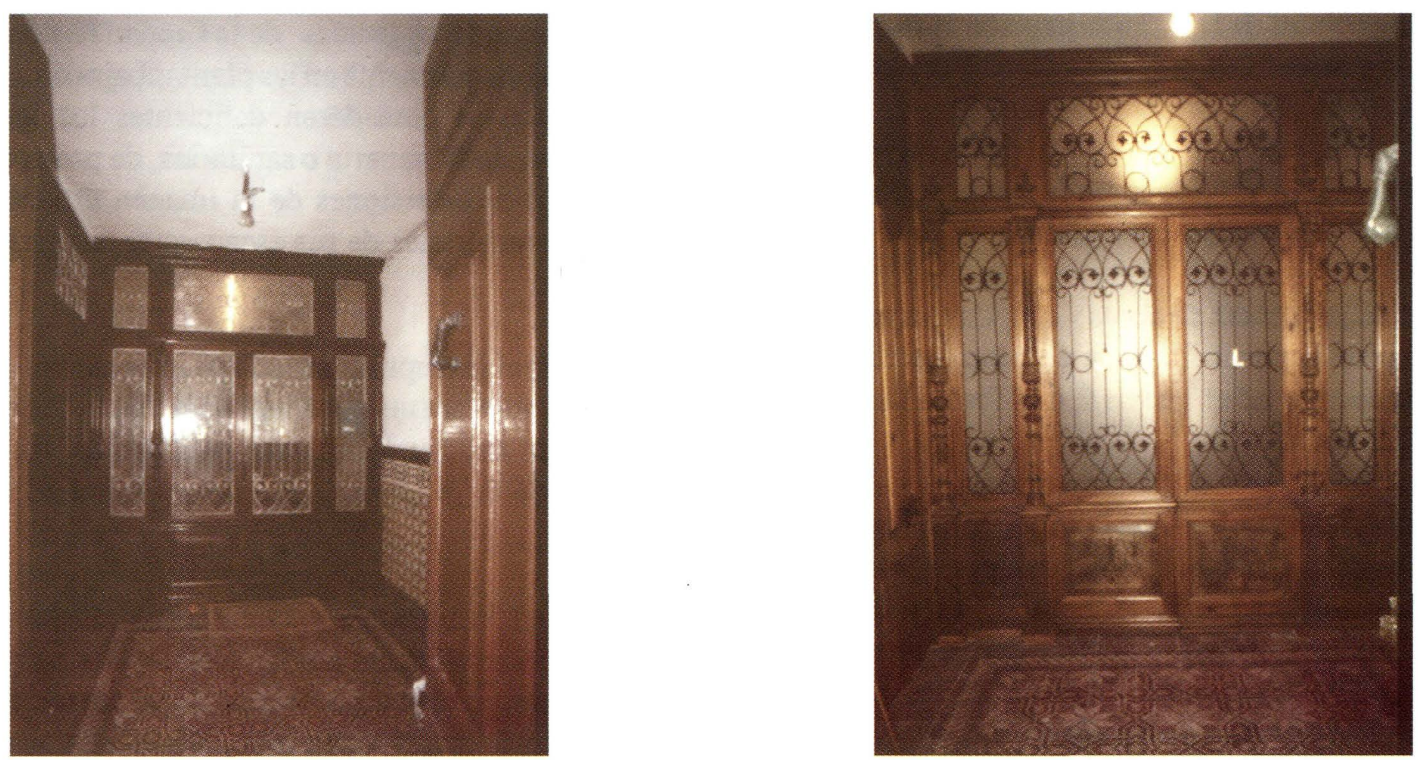

Además de las obras en el interior de las viviendas, el "Plan Puente" contempla la realización de obras en los elementos comunes de los inmuebles. En este caso se acondicionaron las acometidas de agua y electricidad, realizando la centralización de contadores, de agua y eléctricos, se instaló un portero automático y se restauró la puerta interior del portal que figura en las fotografias en su estado inicial y final. En esta puerta, reătizada en madera de pino oregón y castaño, se eliminaron los viejos y degradados barnices y tintes por raspillado superficial, sin recurrir al soplete, y se trató la madera con aceite de linaza y acabado a la cera natural. 
a su vez la documentación requerida, referente a la propiedad de su vivienda o autorización de la misma para realizar obras, declaración de la renta y DNI.

4. Desde ese momento el expediente quedabajo la tutela del arquitecto coordinador de proyectos correspondiente, quien realiza una memoria valorada de las obras solicitadas con los levantamientos de planos necesarios, y cuyo presupuesto es elaborado con el Cuadro de Precios confeccionado por la Oficina para este tipo de obras. Es sobre este presupuesto sobre el que se define el importe de la subvención.

5. Previa aceptación por el particular de las condiciones y presupuestos contenidos en la memoria valorada, la Oficina remite a la Comisión de Gobierno Municipal la solicitud, en la que se pide también la subvención correspondiente al $100 \%$ de las tasas de licencia e impuestos derivados de la realización de obras.

6. Si la subvención es inicialmente concedida, la Oficina tramita la solicitud de licencia de obras del particular, al tiempo que le entrega un ejemplar de la memoria valorada con el que el solicitante puede requerir presupuestos a las empresas constructoras de su elección.

7. Para la elección de la empresa que acometerá las obras, el solicitante puede requerir de la Oficina la relación de Empresas Homologadas por el Concello para este Programa y seleccionar una entre ellas, o bien dirigirse a una empresa cualquiera de su libre elección. Las Empresas Homologadas por el Concello, mediante convocatoria efectuada a comienzos de 1995, se comprometen a aceptar los presupuestos elaborados por la Oficina con carácter de máximos, y cobran de la Administración el importe de la subvención concedida una vez rematada y recibida la obra por los técnicos de la Oficina. De esta forma, y si se ha elegido una Empresa Homologada, el particular sólo pagará el importe de la cantidad que le corresponde una vez descontada la subvención concedida.

8. Concedida la licencia de obras y elegida una empresa, el contrato entre el particular y la empresa se firma en la Oficina, momento en el que se fija la forma de pago y el inicio de las obras.

9. Las obras se desarrollan con la supervisión constante de los técnicos de la Ofícina, que velarán por el correcto uso de los materiales y cumplimiento de las especificaciones recogidas en la memoria valorada por ellos redactada. Todas las observaciones que a juicio de los mismos se hagan en el transcurso de la obra, son recogidas en el "Cuaderno de obra", de cuyo contenido se entrega una copia al encargado de la misma.

Si durante el transcurso de las mismas aparecen daños en la estructura que conviertan la obra en "obra mayor", el solicitante deberá requerir del Colegio de Arquitectos un técnico que se haga cargo de la redacción del proyecto correspondiente, con el fin de poder proseguir la ejecución de la misma.

10. Finalizada la obra y si procede, el arquitecto coordinador de la misma informa favorablemente de su realización y la Oficina solicita de la Comisión de Gobierno la concesión definitiva de la ayuda concedida.

11. Las subvenciones se abonan en el plazo máximo de treinta días a partir de la fecha de concesión definitiva de la ayuda. Para el abono de la parte correspondiente al solicitante, el Consorcio de la Ciudad ha firmado cinco convenios con distintas entidades bancarias mediante los cuales, éstas, facilitan a los usuarios que lo soliciten préstamos personales a un interés moderado para atender la rehabilitación de sus viviendas.

Pese a su aparente complejidad, este sistema evita las desviaciones producidas por presupuestos incorrectos 0 voluntariamente mayorados, a la vez que encauza las obras en la dirección en que aquéllas son útiles a la conservación de este Patrimonio, fin para las que fueron creadas, a la vez que mejoran las condiciones de vida de los usuarios de la Ciudad Histórica.

\subsection{El programa de ayudas a los locales de la ciudad histórica}

El Pleno de la Corporación Municipal, en sesión del día 28 de abril de 1995, aprueba inicialmente la ordenanza de "Ayudas a los Locales de la Ciudad Histórica", presentada por la Oficina de Rehabilitación, con el fin de mejorar las condiciones de los locales de la Ciudad Histórica instalados fundamentalmente en las plantas bajas de los edificios, en los que se consideren deficientes las instalaciones eléctricas, de fontanería o sanitarias, de protección contra incendios o distorsiones de la imagen urbana debido al estado o materiales de sus escaparates, accesos, rótulos o fachadas.

Superado el preceptivo período de información pública sin que se presentara ninguna reclamación, la Ordenanza está pendiente de su inminente publicación en el Boletín Oficial de la Provincia, que determinará su entrada en vigor.

Basada en el mismo sistema de control del uso de las subvenciones públicas que las establecidas para el Programa Puente, pueden acceder a estas ayudas los propietarios o arrendatarios de locales incluidos en alguno de los usos que figuran como subvencionables en la Ordenanza.

Como característica singular es preciso señalar las condiciones recogidas en la misma para poder acceder a las subvenciones y por las que se señalan las obras que deben efectuarse con carácter prioritario. 


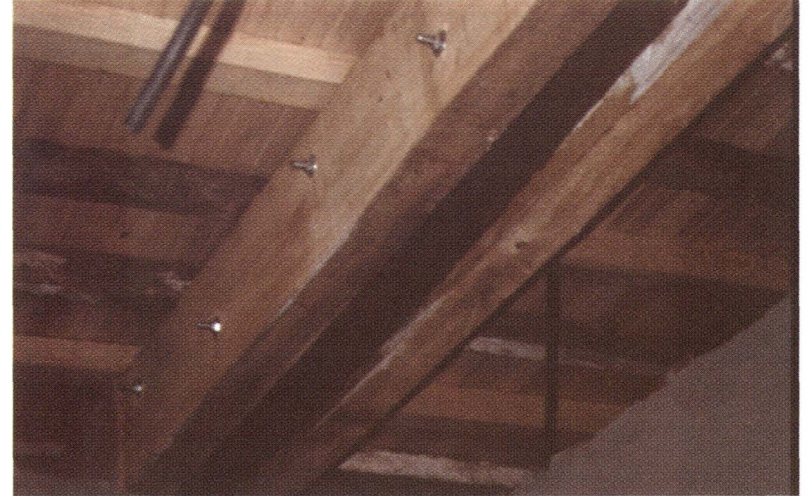

A

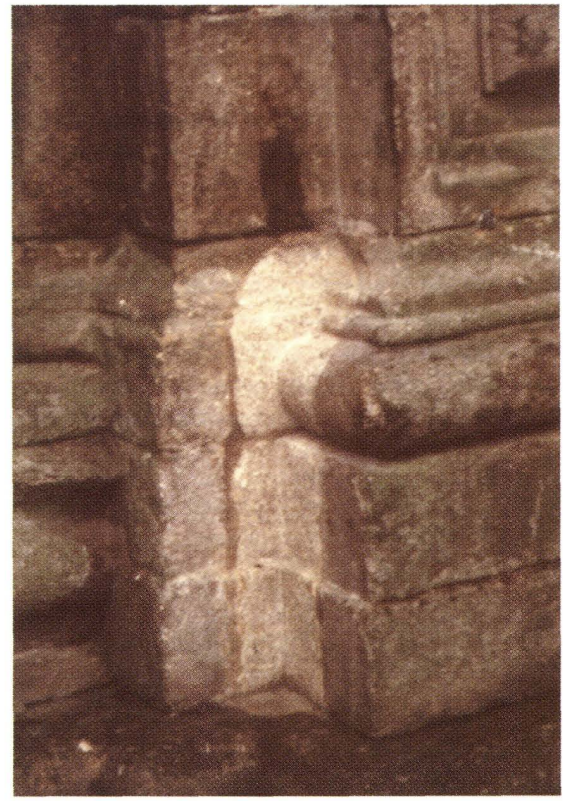

$B$

Se consideran prioritarias la mejora de las instalaciones eléctricas -potenciales causas de incendio en los inmueblesy la mejora de los elementos de fachada ( rótulos, materiales de escaparate, etc.) si éstos no están acordes con el entorno o la normativa vigente.

\subsection{El plan piloto de rehabilitación}

Se agrupan en este Plan el conjunto de iniciativas tomadas desde la Administración para apoyar, complementar, fomentar y desarrollar la pluralidad de estrategias de intervención en el tejido de la Ciudad histórica que los objetivos de una política integral de Rehabilitación requiere.

Las iniciativas, medidas y programas que se desarrollan en el marco del Plan Piloto están incluidas en alguno de los siguientes apartados:

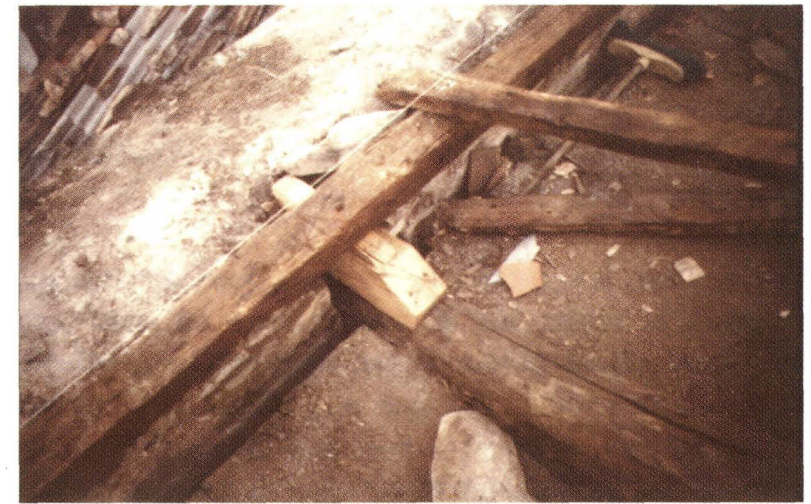

C

En función de la contrastada calidad de los materiales utilizados en la construcción del caserio histórico compostelano, las actuaciones pretenden siempre abordar los problemas con la mayor economía de medios y recurriendo al uso de estos mismos materiales tradicionales. A: Refuerzo del apoyo deteriorado de una viga de castaño mediante el encolado en obra de dos tablones de castaño, afianzados con pasadores de acero inoxidable. Complementariamente se eliminó el origen de la humedad que causó la pudrición. B: Restauración de una basa que había sido cajeada para pasar una bajante de aguas pluviales de la cubierta de la Casa de la Conga en la plaza de la Quintana.Una vez trazada la bajante por un lugar más conveniente, se eliminaron los morteros y se regularizó el cajeado para recibir una pieza de granito que se labró en el sitio para dar continuidad al moldurado. C: El taco de madera de castaño permite afianzar el ensamble de un par de una tijera sobre su tirante y dar apoyo al durmiente, con lo que se consiguió evitar el desarmado de esazona de la cubierta.
- Medidas complementarias de las ayudas a la rehabilitación privada.

- Investigación, difusión y fomento de la rehabilitación.

- Conservación y mejora de las infraestructuras sociales y culturales.

- Programas de reactivación económica.

- Programas de preservación de contenidos funcionales y sociales.

- Programas de conservación del Patrimonio Cultural.

\section{Los usuarios}

Aun cuando es el fuerte carácter de la subvenciones concedidas la condición más apreciada entre los usuarios de las mismas, el papel desempeñado por los técnicos de la Oficina en sus labores de información, asesoramiento técnico y control de la ejecución de las obras es objeto de 
extrema consideración por los solicitantes de estas subvenciones. Agradecen el papel mediador en los innumerables y pequeños conflictos que inevitablemente surgen en cada obra, y valoran especialmente el seguimiento cualificado de las mismas durante su desarrollo.

Por otro lado, la redacción de las memorias valoradas, que incluyen la confección de presupuestos en cada obra, asegura al solicitante una referencia clara sobre el importe de las mismas a la vez que el sistema de Empresas Homologadas, utilizado o puesto a disposición de los particulares, garantiza la realización de las mismas bajo dichos presupuestos.

El trato cotidiano con los solicitantes de estas ayudas ha definido algunas pautas fundamentales en el desarrollo del trabajo de la Oficina.

La Oficina permanece abierta en horario de mañana y tarde, con objeto de facilitar el acceso de los ciudadanos a sus servicios.

Asimismo, se ha constatado entre éstos una evidente falta de información sobre la importancia de la conservación de su ciudad, y sobre las características y cualidades tradicionales de los materiales usados en sus edificaciones.

La aspiración de cambio de estructuras de madera por forjados de hormigón, o la sustitución - ge ne ral mente clandestina- de ventanas de madera por otras de aluminio o PVC que nada tienen que ver con el carácter de su Ciudad Histórica, sin ser generalizada, es una constante que debe ser encauzada desde programas de educación y sensibilización como los que la Oficina diseña en la actualidad.

Mientras tanto, es en cierto modo la labor de información realizada por los técnicos de la Ofícina el medio de contrarrestar esta tendencia, y son las subvenciones establecidas el medio de lograr que éstas sean, a su vez, un argumento de indudable valor en la discusión cotidiana sobre estos temas.

El trabajo desarrollado por el Gestor de la Oficina facilita a los usuarios el asesoramiento preciso en cuestiones de procedimiento administrativo, aclaración de problemas derivados de la titularidad de los inmuebles, y cuanta información precisan sobre otras ayudas a la rehabilitación vigentes y dependientes de otros organismos, tanto autonómicos como estatales.

Asimismo, se ha logrado agilizar la tramitación de los expedientes en el continuo seguimiento y contacto cotidiano con los servicios municipales.

\section{Los técnicos}

Conscientes de que la Oficina de Rehabilitación debe ser el organismo que organice, supervise y coordine la rehabili-

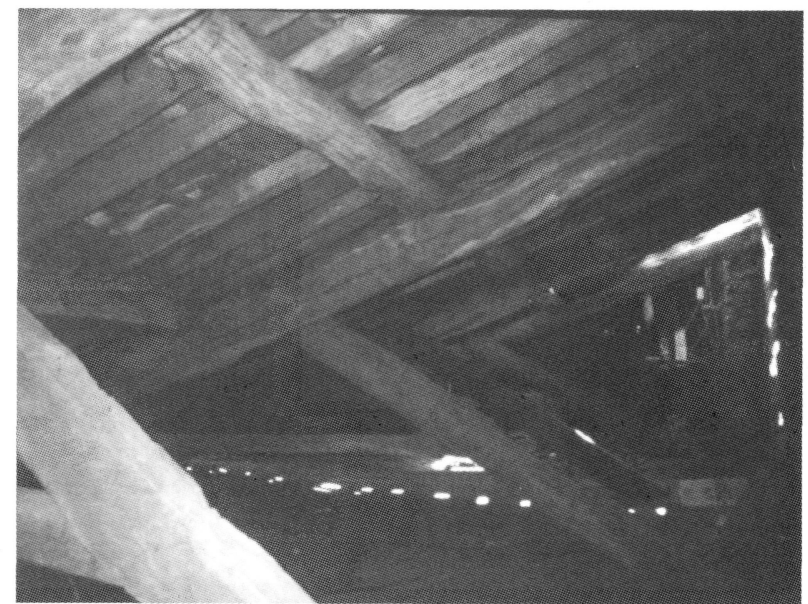

Las cubiertas estan armadas con formas de madera de castaño del pais. Debido a las extraordinarias características de esta madera, y a pesar de la falta de mantenimiento, las patologías observadas no son importantes y cuando se presentan estan muylocalizadas. Sin dudala ventilación que caracteriza estos espacios bajo cubierta, aqui denominados "fallados", es una de las razones del buen estado de conservación de estas estructuras. Con la obsesión contemporánea por acondicionar estos espacios para aumentar la superficie útil de la vivienda inferior, no sólo se pierde un ámbito singular del "alma" de la casa, sino que también se elimina un elemento de gran importancia para el acondicionamiento térmico del edificio.

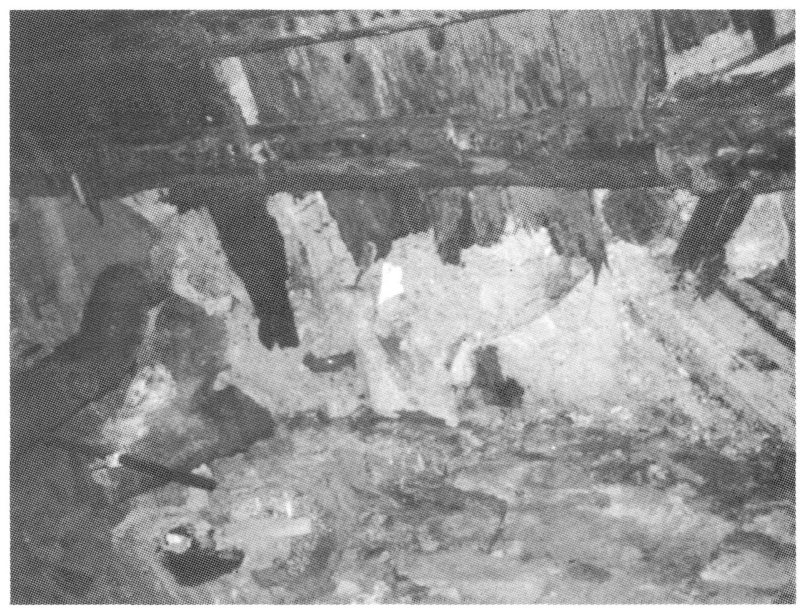

En demasiadas ocasiones la desconfianza de los usuarios de estas viviendas hacia la rehabilitación es debida a la falta de control y deficiente ejecución con que se realizaron este tipo de obras. Esta

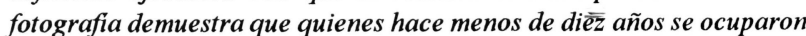
de "rehabilitar" este baño, estaban más preocupados del aspecto de los alicatados, las griferías y los aparatos sanitarios que de impedir la permanente filtración de humedad a la base estructural de madera. 
tación de la Ciudad Histórica, pero en la que deben ser los agentes sociales (usuarios, empresas, técnicos) implicados en su desarrollo y evolución quienes lleven a cabo la Rehabilitación integral de la misma, una vez superada la etapa en la que fue preciso abordar todas las tareas con los medios con los que la Oficina cuenta para poder unificar los criterios de actuación, y conocido ya el alcance de los Programas vigentes, se ha solicitado la colaboración de los técnicos arquitectos residentes en Santiago o que deseen participar en la Rehabilitación de la Ciudad.

Mediante convocatoria la Oficina de Rehabilitación ha reunido cuarenta y ocho arquitectos colaboradores, en su mayoría jóvenes recién titulados interesados en los temas de Rehabilitación.

Mediante contrato puntual de "Asistencia técnica para la colaboración en la redacción y supervisión de obra" en cada caso, estos arquitectos comienzan su actividad profesional al lado de los arquitectos coordinadores de la Oficina, colaborando en el levantamiento de los planos, imaginando las soluciones necesarias y contrastando sus opiniones con los técnicos de la Oficina, quienes asesoran sobre los materiales y soluciones constructivas que forman el criterio de intervención de la misma.

Con estas colaboraciones es posible el desarrollo de las solicitudes presentadas al ritmo que las empresas desarrollan la ejecución de los trabajos, y se consigue dotar a la comunidad de un cuerpo de profesionales especializados para intervenir sobre estas construcciones en el futuro, ya que la restauración y rehabilitación de edificios antiguos sigue siendo una asignatura inexistente en las Escuelas Técnicas, y sólo algunos cursos de postgrado o "masters" cubren este vacío en la actualidad.

Los dos arquitectos coordinadores de la Oficina son los encargados de supervisar el trabajo de los colaboradores, de dirigir redacción de las memorias valoradas y supervisar y efectuar los controles periódicos de las obras.

Paralelamente, la Oficina de Rehabilitación programa y organiza diversos cursos sobre el uso de los materiales en la construcción, con el objetivo sensibilizar y fomentar el uso de las tecnologías y materiales tradicionales entre los trabajadores de las empresas que ejecutan este tipo de intervenciones.

\section{Las empresas}

Ante un Programa de Rehabilitación como el impulsado por el Consorcio de la Ciudad existía la posibilidad, en una ciudad de pequeño tamaño como Santiago, de que el aumento de la demanda de particulares que pretendieran realizar obras al amparo de este Programa produjera un alza de los precios en la ejecución de las obras.
Con objeto de controlar dicha posibilidad, en cierta medida, y asegurar una correcta ejecución de las obras al mismo tiempo, la Oficina propuso al Concello la convocatoria de Empresas Homologadas colaboradoras del Concello en la Rehabilitación interior de viviendas de la Ciudad Histórica.

Dicha convocatoria fue renovada recientemente eliminando alguno de los condicionantes que se exigían en la primera (en concreto, estar en posesión de la Clasificación empresarial Grupo C), siendo en la actualidad treinta y ocho el número de Empresas Homologadas por el Concello para este Programa.

El hecho de "clasificarse" como "Empresa Homologada colaboradora en el Programa de Rehabilitación Interior", presenta para una empresa constructora o un pequeño contratista claras ventajas y determinados condicionantes.

Figurar en un relación de empresas ofrecida por la Oficina para la realización de obras de rehabilitación en las viviendas de la Ciudad Histórica supone un cierto aval y aumento de los contratos de obra, pero al mismo tiempo exige del contratista el compromiso de aceptar (pudiendo ofrecer una baja) los presupuestos elaborados por la Oficina para cada obra, y mantener un aceptable nivel de calidad en la ejecución de las mismas.

La labor desempeñada por los arquitectos coordinadores de obras de la Oficina está logrando encauzar determinados malos hábitos en la ejecución, obligando a rematar con precisión las obras conforme a los detalles constructivos e indicaciones de las memorias técnicas realizadas.

Con el tiempo, y gracias a la supervisión constante de las obras por estos técnicos, se lograrán eliminar ancestrales vicios, fruto de la improvisación y falta de oficio de muchos operadores de la construcción. Dado que la relación con las empresas es constante de una obra a otra, esta insistencia está aumentando la calidad de la obra ejecutada aun a costa de los inevitables inconvenientes que representa una vigilancia constante de la forma correcta de llevar los trabajos.

Las Empresas Homologadas son invitadas a asistir a cuantas conferencias o tertulias organiza la Oficina sobre aspectos puntuales de la construcción, siendo elevado el porcentaje de ellas que asisten a las mismas.

\section{Programas de formación}

En la idea de que sólo con la educación y sensibilización de todos los habitantes y agentes implicados de la Ciudad Histórica, desde el conocimiento de los materiales y las técnicas empleadas en su construcción, se puede mejorar la valoración y respeto del Patrimonio cỡnstruido de Santiago de Compostela, y de que es posible conseguir, en tan sólo 


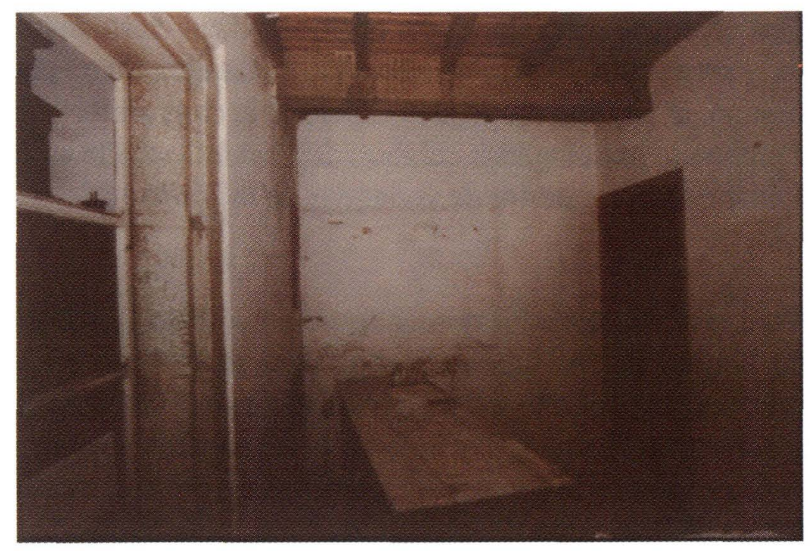

una década, -si se dispone de los medios adecuados para ello- la transformación de la sensibilidad e información actual de los ciudadanos para que la conservación de la Ciudad sea una actividad más y no forzada desde la Administración del Patrimonio, la Oficina de Rehabilitación -con la colaboración de una entidad bancaria asociada al Programa de Rehabilitación mediante convenio firmado con el Consorcio- inició, en marzo de 1995, la programación de una serie de coloquios y conferencias sobre estos temas.

Estos actos, que se realizan periódicamente en las dependencias del Casino de Santiago, según convenio establecido con el Consorcio, están dirigidos a los técnicos y empresas constructoras que trabajan en la Ciudad, siendo la asistencia media a los mismos de cuarenta y cinco personas.

La programación no es cerrada, y se realiza en función del

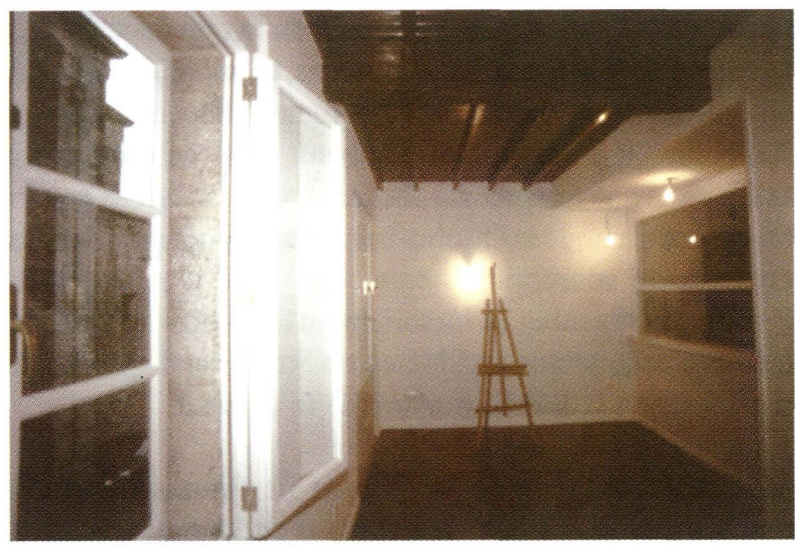

interés y la oportunidad de destacar determinados aspectos de la construcción que permitan, conforme avanza el trabajo de la Oficina, completar la información de los técnicos y empresarios cuya actividad profesional se centra en estos temas.

La entrada a estos actos es gratuita y la difusión de los mismos se realiza con la colaboración del Colegio Oficial de Arquitectos de Galicia.

Actualmente se estudian otros proyectos de colaboración con el Colegio de Arquitectos en este sentido.

En julio de 1995, y con objeto de colaborar en la actualización de las empresas que intervienen en la rehabilitación de la Ciudad, los técnicos de la Ofícina han organizado, en colaboración con la Agencia de Desarrollo Local, un curso práctico sobre "Sistemas de evacuación del agua de lluvia: el cobre y el zinc", financiado por una firma comercial.
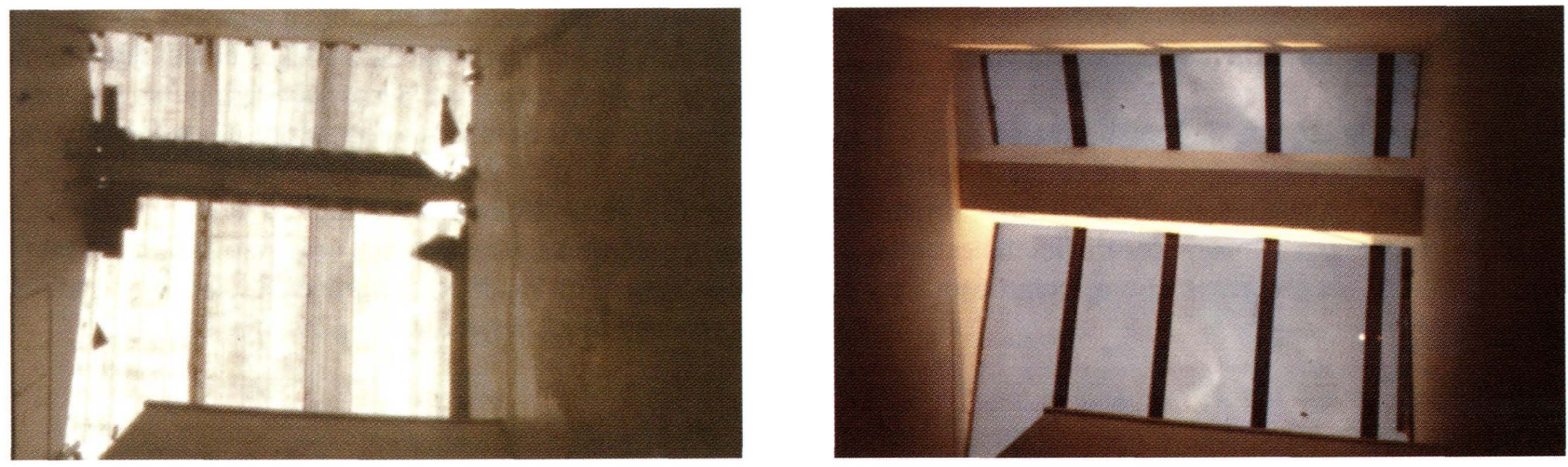

Los tradicionales lucernarios de vidrio que iluminan y ventilan las escaleras se han visto desplazados por las planchas plásticas traslúcidas que en poco tiempo adquieren el aspecto que se observa en la fotografia. Los propios fabricantes de estos productos se refieren a "su comportamiento normal durante 10 años". Esta precisión, más allá de la responsabilidad decenal que tanto nos inquieta a los técnicos hoy en día, debería ser razón suficiente para desestimar su uso en conjuntos construidos con varios siglos de "comportamiento normal". Por otra parte, el avance de latecnología del vidrio nos ofrece hoy día productos que son más estables, más resistentes, más aislantes y que, además, envejecen más dignamente. En las fotografias éstado inicial y reformado de un lucernario de escalera en un edificio construido hace unos 40 años. 


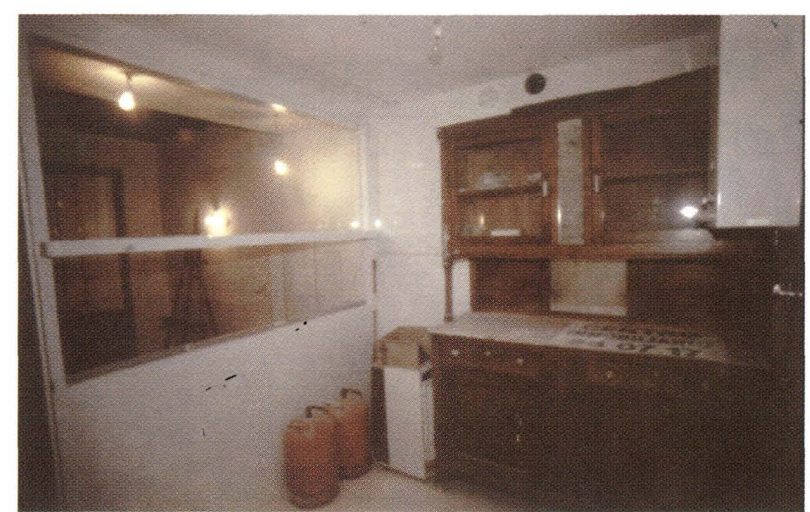

Dirigido a mejorar los conocimientos de oficiales y encargados de obra de las empresas constructoras del entorno, contó con la asistencia de 43 profesionales.

Como resultado de esta experiencia, se encuentran en preparación otros cursos prácticos sobre "Usos y tecnología de la madera en la construcción", "Revocos tradicionales" y "Albañilería en seco", que serán desarrollados en los próximos meses.

\section{Programas de educación}

La inversión pública que supone el mantenimiento de la Ofícina y los presupuestos empleados en los Programas de Rehabilitación aseguran una parte de la conservación de la Ciudad Histórica, aquélla donde se puede llegar mediante las puntuales intervenciones que se efectúan al amparo de estos Programas.

Sin embargo, y para garantizar la conservación de este Patrimonio en el futuro, es preciso completar la labor emprendida con un conjunto de medidas e iniciativas tendentes a mejorar la valoración que de este Patrimonio tienen los habitantes y usuarios del mismo.

Sólo con una planificada y metódica campaña de información sobre el uso de los materiales y elementos constructivos que han hecho a este lugar merecedor de la declaración de Patrimonio de la Humanidad se puede obtener la seguridad de que este Patrimonio será conservado por los propios usuarios en el futuro, pues de ellos dependerá, una vez finalizados los Programas de Rehabilitación, la iniciativa e inversiones necesarias para procurar su conservación.

En los innumerables encuentros mantenidos con los solicitantes de las ayudas establecidas en estos Programas, se ha podido comprobar que en la mayoría de los casos es sólo la falta de información - y no otros factores- los que

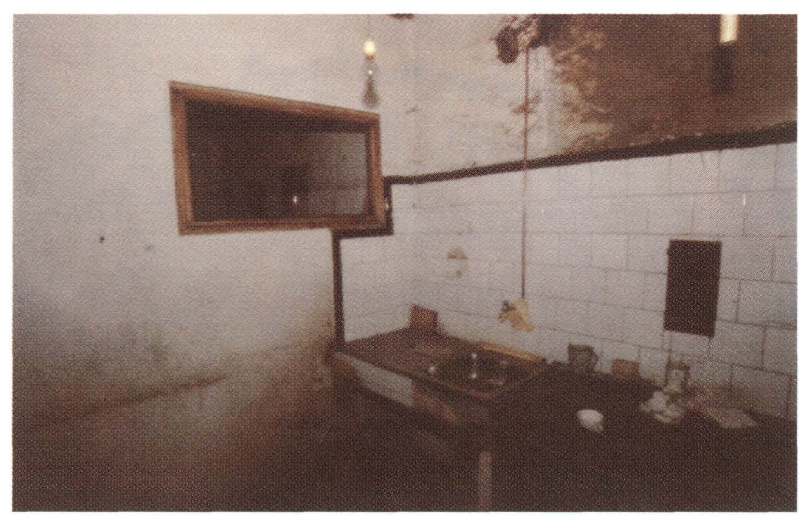

alimentan las reticencias hacia los materiales tradicionales que componen la arquitectura de esta Ciudad.
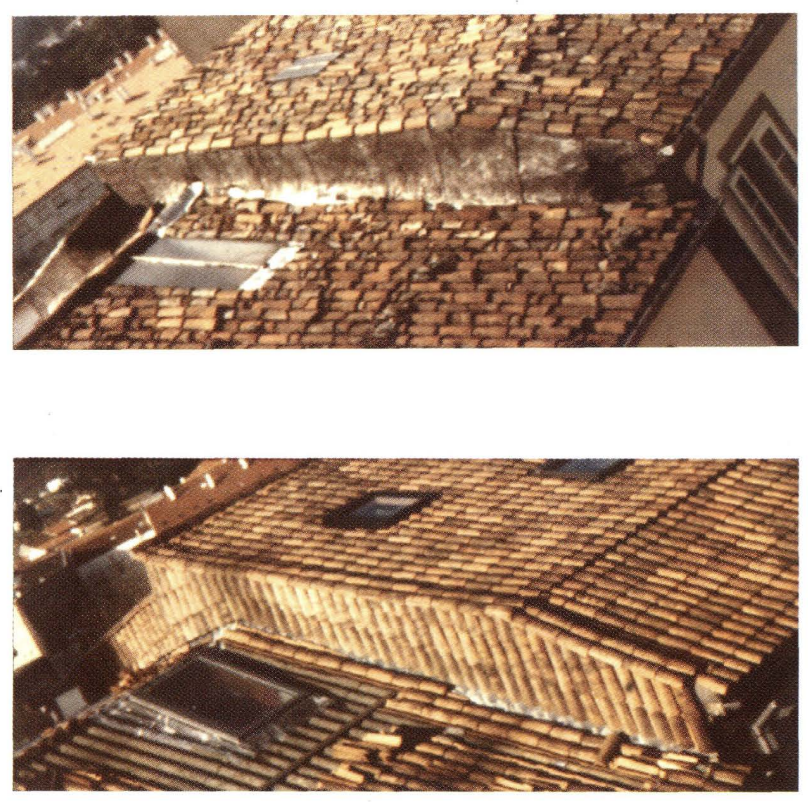

La proliferación de las láminas impermeabilizantes recubiertas de aluminio para solucionar los frecuentes problemas de penetración de agua en medianeras y chimeneas es sin lugar a dudas una solución técnicamente nefasta. A pesar de la aparente eficacia y rapidez con la que quienes no tienen oficio instalan estas láminas a golpe de soplete, los problemas se reproducen o incluso se agravan en poco tiempo. Tradicionalmente los albañiles compostelanos aplacaban con tejas y mucho oficio las medianeras especialmente expuestas a la acción combinada agua-viento. 


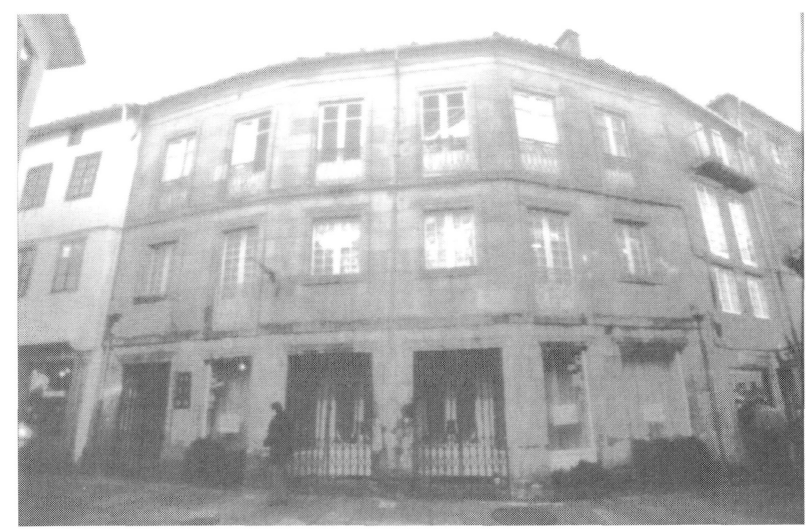

A

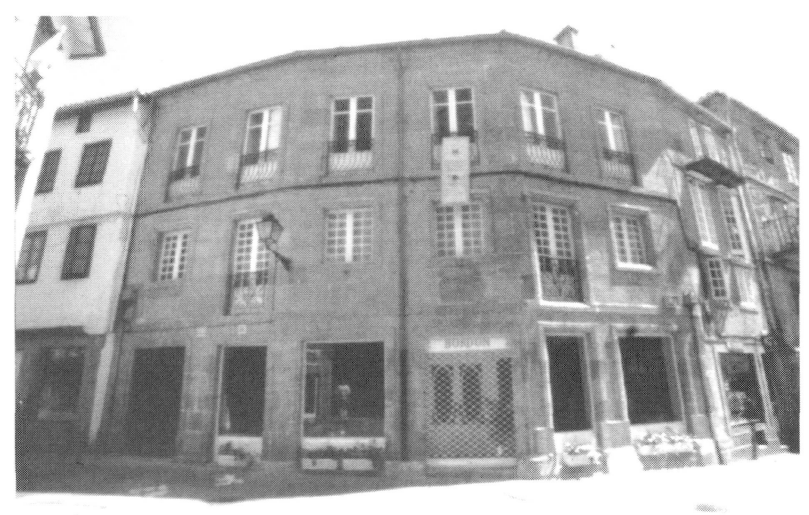

$B$

A y B: Como obras complementarias a la rehabilitación interior de las dos viviendas de esta casa de la rúa do Vilar 81 , se limpió la fachada con agua tibia y cepillo de cerdas, se rejuntó la fábrica con mortero de cal ligeramente coloreado con tierras naturales y se pintaron todas las carpinterías exteriores. También se rehabilitó la cubierta y se instalaron nuevos canalones y bajantes de zinc-titanio prepatinado, desempotrando el pie de bajante y protegiéndolo con un guardacaños de fundición.

La sensibilización actual puede variar radicalmente, si se instrumentan las medidas que pueden hacer comprender la importancia de todo lo que ahora poseemos para el futuro de la ciudad, pues el uso indiscriminado de los nuevos materiales existentes en el mercado no significa, necesariamente, una mejora cualitativa de las condiciones en que se hallan estos edificios.

Sin olvidar ninguno de los avances tecnológicos que procuren una mejora real en la calidad de vida de los habitantes de la Ciudad Histórica o en el mantenimiento de sus edificios, es el cuidado y conservación de lo existente el único camino capaz de garantizar las condiciones arquitectónicas y constructivas que motivaron su declaración como Patrimonio de la Humanidad.

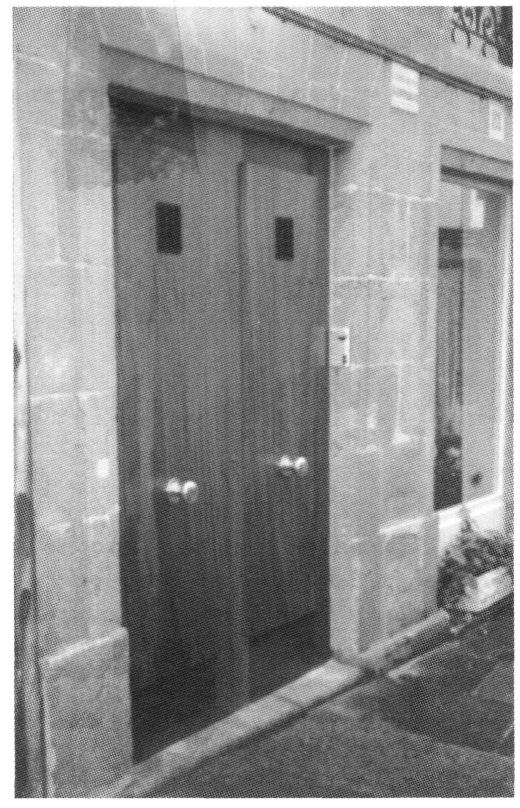

C

(c) Consejo Superior de Investigaciones Científicas Licencia Creative Commons 3.0 España (by-nc)
Con objeto de acercar a los ciudadanos toda la información posible sobre el uso, el mantenimiento con los procedimientos actuales, y las características de estos materiales -durante siglos experimentados con éxito contrastado por los constructores de esta ciudad-, así como de las diferentes soluciones que existen para mejorar su funcionalidad, la Oficina de Rehabilitación prepara una campaña de difusión en colaboración con los centros educativos y otros colectivos ciudadanos.

Otras iniciativas en estudio, en colaboración con el Colegio de Arquitectos de Galicia, intentan aproximar la realidad de Compostela, la importancia de su Patrimonio y de los elementos que lo componen a estos colectivos, mediante conferencias y medidas de difusión gráfica de estos temas.

\section{Alcance del programa puente de rehabilitación interior}

Cuando ya ha pasado un año desde que se puso en marcha el pasado mes de noviembre de 1994 el Programa Puente de Rehabilitación interior de viviendas de la Ciudad Histórica de Santiago de Compostela, los habitantes del Casco Histórico de Santiago han realizado en la Oficina 1.125 solicitudes de visita técnica a edificios o viviendas. De todas estas solicitudes ya han sido realizadas por los técnicos de la Oficina 1.100 visitas de inspección.

C: Detalle de la puerta del portal que finalmente sē instaló en el hueco extremo de la izquierda en esta misma casa de larúa do Vilar 81. Las placas de los porteros automáticos que se instalan son siempre de superficie para evitar el cajeado de las jambas de cantería. 
Como resultado de las visitas técnicas efectuadas, y en función de las recomendaciones del personal de la Ofícina, los ciudadanos han confirmado su intención de acometer obras realizando 670 solicitudes definitivas de subvención.

De estas 670 obras en potencia, la Oficina ha formalizado 275 memorias valoradas, en las que se incluyen planos de estado actual y reformado, detalles constructivos singulares cuando procede, memoria descriptiva de la obra, pliego de condiciones de ejecución y medición y presupuesto de las obras.

El presupuesto total de contrata de las 275 obras documentadas es de 725.000.000 ptas., lo que supone un importe medio por obra de aproximadamente 2.600 .000 ptas. El importe total de las subvenciones para estas obras es de 264.739.711 ptas., resultando un porcentaje medio de subvención por obra del $36,5 \%$ de su coste de contrata con el I.V.A. incluido.

Hasta diciembre de 1995, el Ayuntamiento de Santiago ha concedido 220 licencias de obras, 150 obras se encuentran en diferentes fases de ejecución y 70 obras ya han finalizado.

La experiencia de este año de trabajo en la Oficina Municipal de Conservación y Rehabilitación de la Ciudad Histórica, cuando el proceso de funcionamiento del Programa Puente se puede considerar ya consolidado en todas sus fases, nos permite contemplar con optimismo el incipiente proceso de rehabilitación del extraordinario Con- junto Histórico Edificado de Santiago de Compostela. Y esto es así porque, cada día, un habitante del Casco Histórico decide incorporarse al Programa, cada día se contrata una nueva obra de rehabilitación, $y$, fundamentalmente, porque cada día se termina una obra, lo que significa que las condiciones de habitabilidad de las viviendas mejoran y muchas de esas viviendas, hasta entonces abandonadas, vuelven a ser habitables. Ese es nuestro compromiso.

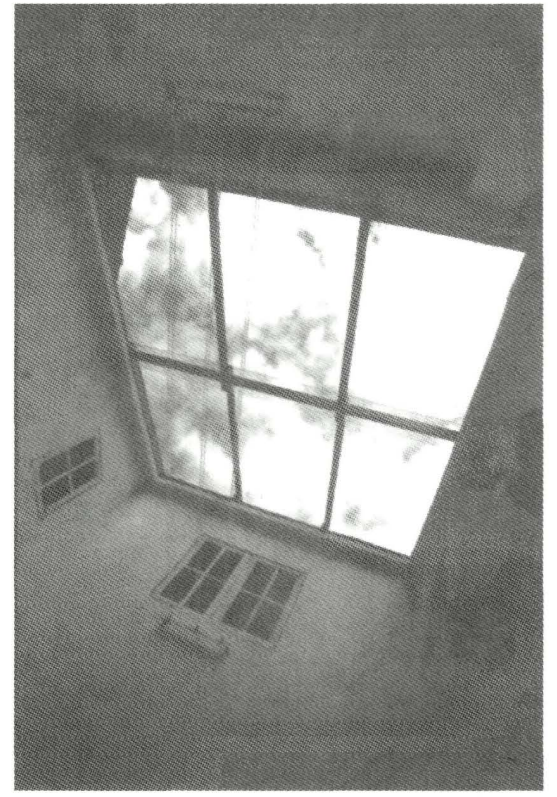

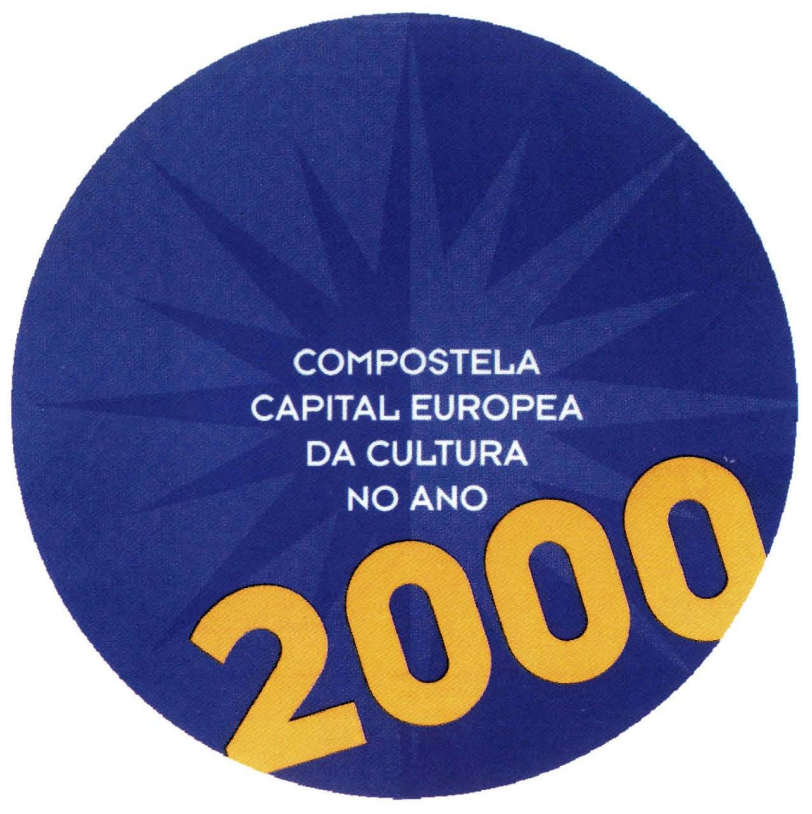

